\title{
eHealth Key Issues in Portuguese Public Hospitals
}

\author{
Ricardo Cruz-Correia \\ Fac. Medicina Universidade Porto \\ (CIDES / CINTESIS), \\ Al. Prof. Hernani Monteiro, \\ Porto, Portugal \\ rcorreia@med.up.pt
}

\author{
José Carlos Nascimento, \\ Rui Dinis Sousa \\ Algoritmi Centre, Information Systems \\ Department, \\ Universidade do Minho \\ Campus de Azurem, \\ Guimarães, Portugal \\ \{jcn,rds\}@dsi.uminho.pt
}

\author{
Henrique O`Neill \\ Instituto Universitário de Lisboa \\ (ISCTE-IUL), ADETTI-IUL, \\ $A v^{\mathrm{a}}$ das Forças Armadas, \\ Lisboa, Portugal \\ henrique.oneill@iscte.pt
}

\begin{abstract}
Hospital managers, health and IT Professionals have their work increasingly supported and dependent on information systems and technologies. To be more effective and efficient in the way health care is delivered, considerable improvements in health information systems and technologies still have to be made at the local and national levels. This is a study that seeks for a deeper understanding of the Portuguese hospitals main stakeholder's concerns regarding their interaction with Information Technology, so opportunities for improvement can be later identified. Seven public hospitals have been selected to participate in this study. In each hospital, managers, IT and health professional were interviewed leading to the discussion of some global and shared perceptions that resulted in a list of eHealth key issues in public Portuguese hospitals.
\end{abstract}

\section{Introduction}

At the turn of the century, it was difficult in Portugal to devise nationwide Health Information Systems. IS solutions deployed in each Hospital or primary care unit were mainly devoted to support locally performed operational tasks and implemented without an integrated perspective. Even when provided by central administration, these solutions were short on perspectives about national integration, the value of data for healthcare management or the relevance of information for clinical decision-making. If this sounds strange in present times, it reflected the health services model of that moment: each unit proceeded as an isolated provider, solving most of their own problems in an autonomous way.

\subsection{Change of national paradigm}

In the last decade, health services' organization experienced significant changes and enormous pressure was set on the IS side. Major opportunities echoed a new paradigm that tends to put the citizen at the center of the health system, demanding for wider perspectives and for organizational models that foster integrated health networks and discourage isolated health units.

\subsection{Challenges at the hospital level}

The pressure to improve the efficiency of the health organizations requires better understanding, communication and coordination of all stakeholders [14]. Therefore, new tools are needed to support innovation and to facilitate improving existing management and working processes. We are now in an period that requires (1) trans-national HIS-strategies, (2) to explore new trans-institutional HIS architectural styles and (3) education in health informatics and/or biomedical informatics, including appropriate knowledge and skills on HIS [5].

Despite the positive effects of Electronic Patient Records (EPR) usage in medical practices, the adoption rate of such systems is still low and meets resistance from some sectors of healthcare professionals. The barriers perceived by physicians to the adoption of EPRs are many and diverse [6].

In hospitals, information technologies tend to combine different modules or subsystems, following a best-of-breed approach [7]. Integration of healthcare Information Systems (IS) is essential to improve health services in hospitals by using scarce resources in a better way, to provide proper care to mobile individuals and to make regional healthcare systems more efficient. However, to integrate clinical IS in such a way that communication and data use for 
healthcare delivery, or research and management, will improve requires many issues to be addressed in a different way [8-10]. Information Systems have evolved to meet people's needs by implementing regional networks, allowing patient access and integration of an increasing number of patient data items. However many distinct technological solutions coexist to integrate patient data, using different standards and data architectures which may difficult further interoperability [11].

\subsection{Central concern with local problems}

As a result of national and local challenges, hospital managers and professionals need to behave and act in a complex environment, under the influence of multiple trends. Organizational, professional, financial, technological, institutional or social drivers continuously raise new challenges to those in charge of managing and using IT to assure that the hospital provides better health services to the citizens.

Aware of the dimension of the problem, the Portuguese Central Administration for Health Services (ACSS) sponsored a research initiative aiming to better understand the nature and dimension of the challenges that Portuguese hospitals face on the e-health domain. It also wants to identify improvement opportunities in technology usage to assure better access to services, more qualified services and resources optimization.

ACSS is aware of the specific characteristics of the different hospitals belonging to the National Health Service network, that may be critical for the success of renewal initiatives: their geographical location, the populations they serve, their size and level of specialization, the different roles of their healthcare professionals, their IS professionals and their managers [12].

\section{Aim}

Driven by the global goals stated here above, this paper presents a study carried out with a representative group of Portuguese hospitals. It aims to define a set of key issues on health informatics as perceived by the main stakeholders: health professionals, managers and IS/IT professional. In particular, these key issues should describe the main IS/IT related concerns these stakeholders recognize as being critical to address the challenges their institutions face.

This project is a contribution to a wider research initiative. As detailed ahead, it presents findings that being important for their intrinsic value may also be appreciated for the support they provide to the subsequent stages of the research initiative concerning the role of IS in the healthcare improvement process.

\section{Methods}

Since we are looking for a deep understanding of hospital stakeholder's concerns coming from their interaction with health information systems and technology, the ontological and epistemological principles of interpretative research [13] [14] seemed adequate to guide our study.

\subsection{Study design and setting}

The study was conducted in the first semester of 2011 seeking reasonable coverage of the territory and representativeness of public hospitals. According to these criteria, an invitation was sent to the Administration Board of seven selected public hospitals and all agreed to participate in the study:

As representative of university hospitals

- Hospital de São João in Oporto (HSJ)

As representative of district hospitals in interior regions

- Centro Hospitalar Trás-os-Montes e Alto Douro in Vila Real (CHTAD)

- Unidade Local de Saúde da Guarda (ULSG)

As representative of district hospitals in littoral, highly populated regions

- Centro Hospitalar Vila Nova de Gaia (CHVNG);

- Hospital Fernando da Fonseca (HFF)

- Centro Hospitalar de Setúbal (CHSetúbal)

As representative of district hospitals in the south

- Hospital Distrital de Faro (HDF)

\subsection{Participants}

At each hospital, we applied for the participation of two groups of professionals regarding their main role in using or providing health information systems and technology at the public hospitals: health professionals and management and IT professionals. In each group, two main stakeholder representatives were invited to participate:

- Health professionals

○ Clinical director

- Head of nursing

- Management and IT professionals

○ Head of IT department

- Administration board member responsible for IT

\subsection{Data collection}

The perceptions of the main stakeholders in the selected public hospitals were collected using a semistructured type of interview. Given the distinct professional nature of each group of professionals, two 
separate interviews were conducted for each group with the two main stakeholder representatives.

Each interview lasted for about one hour, and was addressed in a fairly open manner to enable the stakeholders to express their thoughts and priorities. Starting by recalling the objectives of the study, the interviewers asked the stakeholder representatives to comment on their concerns, barriers to overcome, or opportunities to seize in order to improve the use of health information systems and technology regarding the following issues [6]:

- Financial constraints

- Technical difficulties

- Time constraints

- Psychological (e.g. attitudes from users/ administration towards technology)

- Social (e.g. relation between IT and health professionals)

- $\quad$ Ethical, security and legal

- Organizational difficulties

- Change process

Written notes were taken during the interviews and, to enable further analysis, all interviews were recorded, after asking for permission and ensuring their confidentiality to the participants. At least two researchers out of a team of four carried out each one of the interviews at the seven public hospitals. To limit for possible bias in the way the stakeholders were interviewed and questions were raised, the first interview was conducted with the participation of the four interviewers.

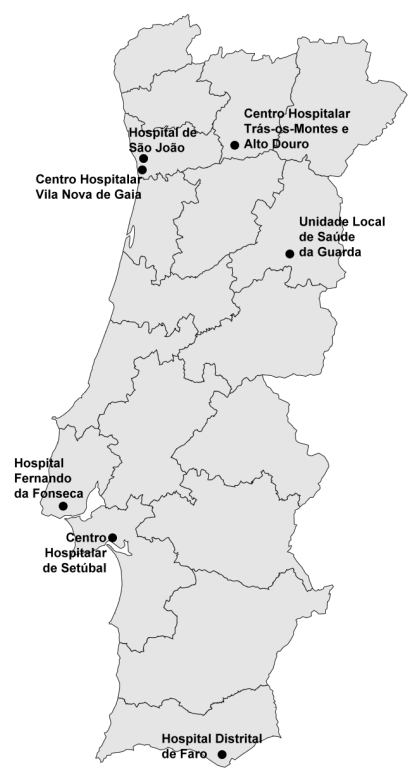

Figure 1. Location of Hospitals

\subsection{Data analysis}

The analysis of the interviews to uncover key issues on health informatics was performed according to the following steps:

- The written notes taken during the interviews were gathered;

- The written notes were reviewed while hearing the recorded interviews;

- A single set of issues stressing the four interviewers' agreements was created.

\section{Results}

\subsection{Global perception from interviews}

A global set of issues emerged spontaneously as a result of the perceived relevance of the topic to the interviewees and due to its persistency across several interviews.

First of all it is clear that doctors and nurses are aware that health services changed significantly in the last decade and their professions are increasingly dependent on information systems and technologies. To make better decisions and provide better services professionals need to access assorted information from different sources, such as medical devices, healthcare diagnostics, prescriptions systems, patient historical information or information made available by other health units and services. Healthcare activities are no longer isolated acts and, as such, health and management professionals are very aware of the growing dependence on effective and accurate IS to support integrated healthcare processes.

\subsubsection{Lack of integration at the Hospital level}

Related to the previously mentioned, many of the clinical directors stated that a growing need to fetch data in different systems, using dissimilar applications and interfaces and getting diverse formats is probably the major difficulty to the efficient accomplishment of everyday tasks. The need to "manually" integrate all the information is shown to be very timing and resources consuming, potentially inaccurate and mostly "non-reusable". The high level of fragmentation, boosted by the lack or incompleteness of interoperability platforms, was identified as an important concern to the health professionals' daily activities and also a demanding challenge to the IT departments.

Due to these difficulties it was not surprising to find that the adoption of EPRs, at the hospital level, emerged as the envisaged solutions to all the interviewees. Furthermore, professionals perceive EPR as the solution to the actual lack on integration, both in Hospitals without any experience and in Hospitals that are ahead in the process of implementation of an EPR. 


\subsubsection{Lack of central leadership and planning}

From the interviews resulted that Management and IT professionals are strongly concerned with the lack of leadership and regulations defined by central authorities. During the last decade, national authorities were challenged by significant changes in health services organization. Nevertheless, no clear answers were given in terms of IS national policies, despite the high expectations that were raised to the role of IT in the health sector. Interviewees stressed that, due to the fact that today's Hospital is no longer an isolated health unit but a member of a network of health services providers, lack of guidance raises serious concerns.

\subsubsection{Unclear model for HIS sourcing}

The sourcing models for health IS solutions are still very unclear. IS core solutions to Public Hospitals and Primary Care units were traditionally provided by the health central administration, at no cost and with a service level based on a "better effort" approach. Today the data integration between public "free" applications and market solutions is not an easy task.

Due to the technical obsolescence of state owned systems and without a real clarification about the IS Solutions sourcing models, selecting a new solution is always a difficult task, due to the high level of uncertainty the IS sourcing processes presents in this context.

\subsubsection{Central authorities get data from hospitals without a "win-win" attitude}

At a different level - but probably with the same underpinning roots - the interviewees expressed a great concern about the process used by central authorities to collect data from hospital, which is generally done in an uncoordinated and even in intrusive way.

Interviewees stated that due to the growing demand for performance indicators and benchmarking data at the higher levels of decision, central authorities tend to enforce web based applications to collect diverse sorts of operational data that is produced at the hospitals level. With a few honorable exceptions, these procedures tend to ignore already existing data and current hospital procedures, depending more on hierarchical power rather than collaboration to the development and implementation processes. In spite of the perceived relevance of nationwide data, all professionals raise serious concerns about how the collecting process is implemented, causing work redundancy, data misalignment and little benefits to the hospital.

\subsection{Key issues}

A more detailed analysis of the interviews leads us to define a clear set of key issues (see Table 1). These key issues were grouped in the following classes:

I. Technical and Administrative issues addressed the suitability of available resources, not only from a technical point of view (computers, networks, applications but also from a financial, technical support and performance points of view.

II. Clinical issues covering problems and concerns that relate with daily clinical activities and the use of health data and applications in the Hospital, including EPR.

III. Integration and Regulation issues, covering more formal aspects of systems integration, business processes, national policies (including standards and certification), global interoperability and integration (including national systems and applications) and role of diverse stakeholders and suppliers in the sourcing process of Health IT solutions.

IV. Professional issues, such as managers and health professionals' awareness to IS problems, literacy in IS issues, commitment and readiness to change, among others.

Table 1. List of identified key-issues grouped by the defined classes

Technical and Administrative

1. IT networks are slow and/or sometimes unavailable.

2. Health professionals are obliged to use too many IT applications on daily activities.

3. Information systems management in the Hospital lacks reliability and maturity.

4. With today's IT solutions it's not easy to produce statistics or to get performance indicators.

5. IT department services are slow or inadequate in view of Hospital needs.

Clinical

6. Health information systems lack integration and/or interoperability.

7. Hospital needs a EPR (Electronic Patient Record) to provide an integrated view of patient information.

8. The co-occurrence of paper and electronic clinical records causes incoherencies and redundant work.

9. With today's systems, confidentiality is not guaranteed in a suitable manner.

10. Health coding and classification systems are rarely or weakly used.

11. IT solutions are tricky to use due to very heterogeneous "look and feel".

12. Today's electronic health records don't support clinical research in a suitable manner.

13. Existing IT solutions are mainly "all-purpose" and don't address clinical specialties.

14. Access to patient health data stored in other health 
institutions is very difficult or even impossible.

15. Some IT health solutions demand too much data and/or useless details.

16. Hospital management has very low awareness about IS importance to Hospital's strategy.

Integration and Regulation

17. IT services provided by health Central administration are scarce and/or inadequate to the Hospital needs.

18. IT services provided by IT companies don't suit Hospital needs.

19. Quality and assortment of eHealth solutions available from IT companies are unsatisfactory.

20. Organizational processes are poorly defined, raising significant difficulties to IT projects.

21. The lack of national adopted standards and IT certification mechanisms defies IT management in Hospitals.

22. Lack of national leadership concerning IT causes uncertainty and trouble to hospital IT management.

23. Web solutions are too often enforced by central authorities, without bearing in mind hospital processes or local existing data.

24. Difficulties and obstacles to the integration of market IT solutions with core public solutions restrains acquisitions.

25. Uniqueness of patient identification and data integration are very problematic tasks due to the lack or insufficient use of national master indexes.

26. The absence of a national EPR raises serious barriers to the development of a citizen-centered health system.

Professional

27. Health professionals are poorly committed to use IT systems.

28. It's difficult to gain health professionals contributions during the specification and deployment of health IT solutions.

29. The ratio between "time spent using IT solutions" and "resulting benefits to health care" is still exceedingly low.

\section{Discussion}

Contributions from this exploratory study clear fit research goals. First, the resulting list of key issues in health informatics provides a solid foundation to subsequent stages of the research initiative under way. Second, relevant preliminary prepositions have emerged reinforcing the need for a national corroboration of the theoretical assumption.

\subsection{Examining the key issues}

The resulting list of key issues in health informatics is in line with the literature and researchers expectations, exception made to some specific aspects that will be discussed ahead.

In fact, the set of key issues shows a good balance with the concerns identified during the literature review, ranging from technical to ethical, including also financial, organizational or professional issues, among others. However, it is important to state that the influence of actual Portuguese eHealth process is well sensed in the final results.

For instance, this research found that immediate and practical issues deserve more attention from professionals than those "softer" issues that deal with ethical or more theoretical aspects. Appearing to be a "maturity problem", it probably relates to the fact that the adoption of new paradigms is yet in early stages and, consequently, professionals tend to focus in basic and operational needs. For instance, easiness of access and process integration are seen as crucial for doctors' day-by-day activities, while issues like data confidentiality, adoption of standards or the need of more structured data are perceived as "important" but not as "immediately critical".

Supporting these propositions, gathered information also expressed that two processes of integration run in parallel in Portugal: a process that deals with the integration at a national level and another process that aims to integrate Hospital's systems and processes. Although global concerns were indicated by all participants, it is clear that internal issues go up in the professionals' priorities, both from health professionals and managers in Hospitals.

Due to the amount of IS problems that need to be solved at hospital level and the huge impact that they have to daily activity, professionals tend to focus on internal problems. As such, external problems, at a national level, earn some awareness - which is good but no real attention or commitment.

\subsection{The non-issues}

In spite of our findings in line with the literature, further discussion is due to some specific topics. In truth, during this stage of the research, despite the alertness of researchers, the interviewed stakeholders raised no special concerns about some issues that are mentioned in literature and that, therefore, where part of researchers' previous referential and expectations

In effect, all health professionals and managers from the seven hospitals expressed no particular concerns regarding issues like health professionals' IT literacy and motivation, scarceness of financials resources or even limitations on available technical resources (except for national health data network).

When mentioned by researchers, these subjects were acknowledged as essential to implement successful health information systems but no interviewee selected any of them as a major issue in his own context. From their understanding, other issues rather than these can be accountable for projects that fail or don't see daylight. 


\subsection{Limitations of this stage}

Even if the number of key issues $(n=29)$ and seniority of participants in this stage were relevant, it is important to bear in mind that only seven Hospitals (from a total of 56) were included in this exploratory research.

Additionally, even if some precautions were taken during Hospital selection to minimize bias, the maturity of the selected group may be above the national average. This maturity, for instance, could be an explanation for the "non-issues" problem that was identified and, as such, further research is required.

Finally, it is important to take note that changes in the eHealth scenario in Portugal are occurring very fast, which gives the interviewed professionals almost no time to acknowledge them and process all the occurring phenomena. Nevertheless, this should be seen not as a limitation but as a feature of the current change process.

\subsection{Research's next steps}

The list of key issues, the brief rationale for each issue and the insights on the problem provide an important framework to the prosecution of a research that aims to develop a national perception on health informatics.

Following this work, a Delphi study will be conducted at a national level, in order to provide a consensual and ranked list of key issues, both from IT and clinical perspectives.

These two perspectives of eHealth key issues in Portuguese Public Hospitals and the subsequent analysis will be relevant to the knowledge in this area and "to identify improvement opportunities in technology usage to assure better access to services, more qualified services and an optimization of resources", as commissioned by the Portuguese authorities.

\section{Acknowledgements}

The authors would like to thank the Central Administration for Health Services (ACSS) of the Portuguese Health Ministry for sponsoring this study.

\section{References}

[1] M. Holland, Young, J., "Improving clinical workflow through effective context and identity management," Health Industry Insights, December 20062006.

[2] J. Becker, Jamiesch, C., "Resctrictions in Process Design: A Case Study on Workflows in Healthcare," in 5th International Conference on BPM, Australia, 2007.

[3] S. Malhotra, Jordan, D., Shortliffe, E., Patel, V., "Workflow modelling in critical care: Piecing together your own puzzle," Journal of Biomedical Informatics, vol. 40, pp. 81-92, April 20072007.

[4] M. Duitshof, "Workflow Automationin Three Administrative Organizations.," Masther's Thesis, Department of Computer Science - Section Information Systems, University of Twente - The Netherlands, Twente, 1995.

[5] R. Haux, "Health information systems -- past, present, future," International Journal of Medical Informatics, vol. 75, pp. 268-281, 2006.

[6] B. Albert and B. Manda, "Barriers to the acceptance of electronic medical records by physicians from systematic review to taxonomy and interventions," $B M C$ Health Services Research, vol. 10, 2010.

[7] R. Lenz and K. A. Kuhn, "Integration of Heterogeneous and Autonomous Systems in Hospitals," Business Briefing: Data management \& Storage Technology, 2002.

[8] H. Heathfield, D. Pitty, and R. Hanka, "Evaluating information technology in health care: barriers and challenges," British medical journal, vol. 316, pp. 19591961, 1998.

[9] M. Berg, "Implementing information systems in health care organizations: myths and challenges," International Journal of Medical Informatics, vol. 64, pp. 143-156, 2001.

[10] P. Littlejohns, J. C. Wyatt, and L. Garvican, "Evaluating computerised health information systems: hard lessons still to be learnt.," British medical journal, vol. 326, pp. 860-3, Apr 2003.

[11] R. J. Cruz-Correia, P. Vieira-Marques, A. Ferreira, F. Almeida, J. Wyatt, and A. Costa-Pereira, "Reviewing the integration of patient data: how systems are evolving in practice to meet patient needs," BMC Medical Informatics and Decision Making, vol. 7, p. 14, 12 Jun 2007.

[12] B. Kaplan, "Addressing organizational issues into the evaluation of medical systems," Journal of the American Medical Informatics Association, vol. 4, p. 94, 1997.

[13] H. K. Klein and M. D. Myers, "A set of principles for conducting and evaluating interpretative field studies in informations systems," MIS Quarterly, vol. 23, p. 67, Mars 19991999.

[14] W. J. Orlikowski and J. J. Baroudi, "Studying Information Technology in Organizations: Research Approaches and assumptions," Information Systems Research, vol. 2, pp. 1-28, 1991. 